\title{
Le plan crue de la RATP
}

\section{The RATP plan of action in case of flood}

\author{
par J. Favreul
}

Régie autonome des transports parisiens

Today, the good working of the public transport is essential for regional economy and population life. More over, a long network stopping costs a lot, as well as the restoring of the fixed equipments after a flood. The RATP has established an action plan which covers the measures to take in order to protect the works. This plan of action, which supposes a flood not exciding that of 1955 , needs to be frequently updated.

\section{LES TRANSPORTS EN COMMUN D'ILE-DE-FRANCE}

\subsection{Complémentarité des modes}

Les réseaux de la RATP, bus, tramway, métro, RER, assurent avec le RER de la SNCF plus des neuf dixièmes de la desserte de la région en transports en commun ; l'arrêt accidentel du trafic, s'il se prolonge, nécessite pour les usagers de la ligne concernée dont ils sont captifs particulièrement aux périodes quotidiennes d'affluence, le détournement de leur itinéraire sinon la recherche d'un mode de transport de remplacement. Si de tels arrêts de trafic visent simultanément plusieurs lignes et qu'ils se prolongent au-delà de la journée, leur complémentarité ayant atteint alors sa limite, les modes de remplacement ne répondent plus aux besoins et l'utilisation excessive des voitures particulières qui en résulte provoque l'obstruction croissante de la voie publique. Cette situation caractérisée par le manque de transports publics et la difficulté voire l'impossibilité de se déplacer par d'autres moyens, affecte dangereusement la vie économique et peut conduire à la paralysie totale des échanges.

\subsection{Les réseaux ferrés parisiens}

En 1910 (fig. 1), sur les sept lignes de métro alors en service (cette figure ne représente pas les cinq autres lignes en construction), cinq lignes dont la partie centrale des tunnels avait été inondée, avaient dû être partiellement fermées au public, car notamment, le mode d'alimentation en courant électrique avait permis d'exploiter des extrémités de lignes. Le manque d'archives ne permet pas de connaître un ordre de grandeur du coût de la remise en état de ce qui était à l'époque un réseau encore modeste et doté d'équipements relativement sommaires. Son indisponibilité n'avait probablement pas dû faire subir aux Parisiens, eu égard au temps perdu, un préjudice trop important.

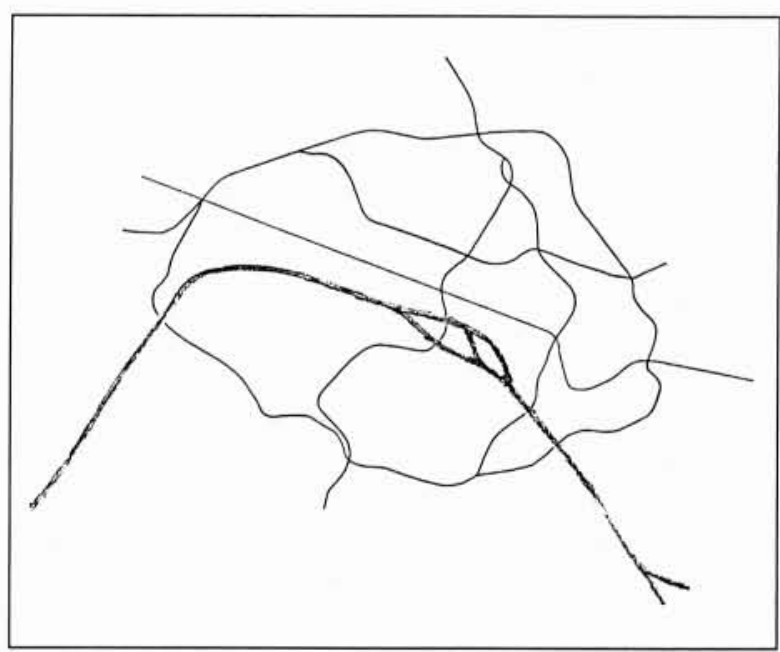

1. Le métro de 1910.

En 1997, l'ensemble du réseau ferré parisien est constitué par 15 lignes de métro, 4 lignes de RER dont les lignes $C$ et D exploitées par la SNCF et la ligne Météor prochainement ouverte au public. La figure 2 est un schéma simplifié du tracé en plan de ces vingt lignes qui à Paris et en proche banlieue sont chacune, à quatre exceptions près, entièrement souterraines. Aucune ligne ne se croise à niveau, mais les tunnels sont en général reliés deux à deux par des voies de service et les lignes sont aussi reliées entre elles par les correspondances et par bien d'autres ouvrages communs, de 


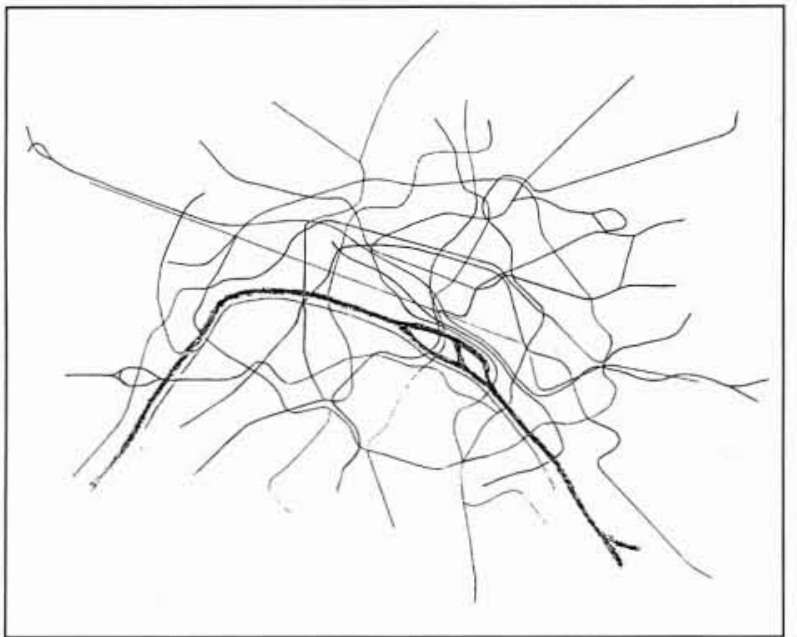

2. Les réseaux ferrés parisiens de 1997.

sorte que tous les ouvrages ferroviaires souterrains constituent un ensemble maillé non seulement eu égard à l'air qui y circule mais aussi eu égard à l'eau qui pourrait s'y déverser en cas d'inondation catastrophique.

Aujourd'hui, le courant électrique nécessaire à la traction ainsi qu'à l'exploitation des gares et stations est fourni par EDF et la marche des trains est contrôlée sur chaque ligne par un poste central. En cas d'incident, même s'il est mineur, le bon fonctionnement des postes centraux qui constituent, comme la permanence générale des réseaux et le PC Sécurité, des systèmes d'information tributaires de réseaux de télétransmission et de télécommunications, est indispensable : la rupture prolongée des liaisons entre la ligne, son poste central et les agents de conduite des trains détermine l'arrêt de l'exploitation, les postes de secours locaux n'ayant été dimensionnés que pour pallier une rupture passagère des liaisons ; bien d'autres équipements fixes dont le bon fonctionnement est indispensable ont été installés pour conjuguer amélioration des performances d'exploitation et accroissement de la sécurité ferroviaire.

Le tableau 1 indique les valeurs comparées d'immobilisations en milliards 1995 .

\begin{tabular}{|l|c|c|}
\hline & Métro $*$ & RER ** \\
\hline Ouvrages & 43 & 21,2 \\
\hline $\begin{array}{l}\text { Ateliers de } \\
\text { maintenance }\end{array}$ & 3,6 & 2,1 \\
\hline Equipements fixes & 6,7 & 5,2 \\
\hline Autres équipements & 3,3 & 1,9 \\
\hline Matériels roulants & 12,4 & 9,1 \\
\hline Totaux & 69 & 39,5 \\
\hline$*$ Ligne Météor exclue $* *$ Lignes A et B RATP \\
\hline
\end{tabular}

Tableau 1. - Valeurs d'immobilisations 1995 en milliards.

On notera particulièrement, à longueur égale de ligne d'une part, le coût nettement plus élevé des ouvrages et équipements du RER, réseau à grand gabarit et plus profondément établi que le métro, et, pour l'ensemble des lignes d'autre part, la fraction relativement importante du coût des équipements fixes qui inclut celui des essais indispensables effectués avant leur mise en service.

Dans les ouvrages souterrains, des drains assurent l'écoulement des inévitables eaux d'infiltration que des pompes ou éjecteurs à débit limité et à fonctionnement intermittent refoulent à l'égout au rythme des dépassements de niveaux dans les puisards. Dans les voies sur ballast, l'envasement progressif des drains avec le temps nécessite leur curage qui, dans certaines configurations, ne peut véritablement être effectué qu'à l'occasion des travaux de renouvellement du ballast ; à la mesure de leur profondeur respective, les ouvrages souterrains modernes, souvent établis dans la nappe phréatique, ont été conçus pour résister tant à la poussée d'Archimède par lestage, qu'à la pression de la nappe dans l'hypothèse de sa remontée. Les fréquentes inégalités de répartition des poussées et des pressions le long des tunnels et des gares souterraines peuvent induire des couples de torsion qui provoquent des fissurations de parois ou de voûtes nécessitant leur colmatage à court terme pour éviter la saturation des éjecteurs ou des postes d'épuisement.

Dans l'état actuel de la recherche, il n'est guère possible de préciser en cas de crue et pour chaque ouvrage, la hauteur limite atteinte par la nappe alluviale au-delà de laquelle l'ouvrage devrait être délibéremment noyé pour éviter sa fissuration et les infiltrations consécutives en de trop nombreux endroits ; en tout état de cause, un ouvrage ne pourrait être ainsi noyé à titre préventif qu'après obstruction étanche de toutes ses communications avec les ouvrages mitoyens à sauvegarder .

\section{II $\square$ LE PLAN CRUE}

\subsection{Vulnérabilités particulières}

En raison de l'intercommunication entre tous les ouvrages souterrains des réseaux ferrés parisiens de la RATP et de la $\mathrm{SNCF}$, au cas où la ligne d'eau atteinte en surface par débordement de tous parapets et barrages par la Seine correspondrait à celle de 1910 , la figure 2 montre que l'étendue des volumes immergés serait maintenant considérable ; en raison de la durée des pompages à effectuer à la décrue, des délais nécessaires à la remise en état des ouvrages, au renouvellement quasi-complet de la plupart des équipements fixes et à leurs essais de bon fonctionnement, une telle catastrophe rendrait indisponibles pendant de nombreux mois les réseaux ferrés parisiens, dont seules les sections de banlieue pourraient continuer d'être exploitées en mode dégradé et sous réserve de leur alimentation par EDF ; le coût global pour la collectivité se chiffrerait en milliards.

Pour le cas du débordement par submersion de tous les parapets, les figures 3 et 4 qui représentent respectivement les niveaux caractéristiques d'ouvrages rive droite dans le secteur de la gare de Lyon et rive gauche dans le secteur Saint-Michel, montrent la difficulté rencontrée pour concevoir les multiples barrages propres à éviter le déversement des eaux dans les ouvrages souterrains et qui devraient être édifiés bien avant que la Seine n'atteigne le niveau de sa crue de 1955.

\subsection{Mesures du plan}

Ces mesures sont énoncées, pour chaque ouvrage concerné, en fonction des niveaux croissants successivement atteints par la Seine ou par la Marne et de la tendance de la crue ; ainsi, il convient de vérifier au préalable le bon fonc- 
Pont

d'Austerlitz NGF

8,6 34,5 Crue de 1910

$7,1 \quad 33 \quad$ Crue de 1955

32,5

Rue de Bercy

Quai de la Rapée

$27 \quad$ Ligne 1

0,8

26,7

25

Salle RER Météor

21 Ligne Météor

20 RER ligne D SNCF

14

13,8 RER ligne A RATP
Parcs en sous-sol

Fond de cuvelage

du siège de la RATP

(Coupe perpendiculaire à la Seine, le sud à droite)

3. Rive droite : secteur de la gare de Lyon.

(Le niveau donné pour une ligne correspond à celui du radier de ses voies).

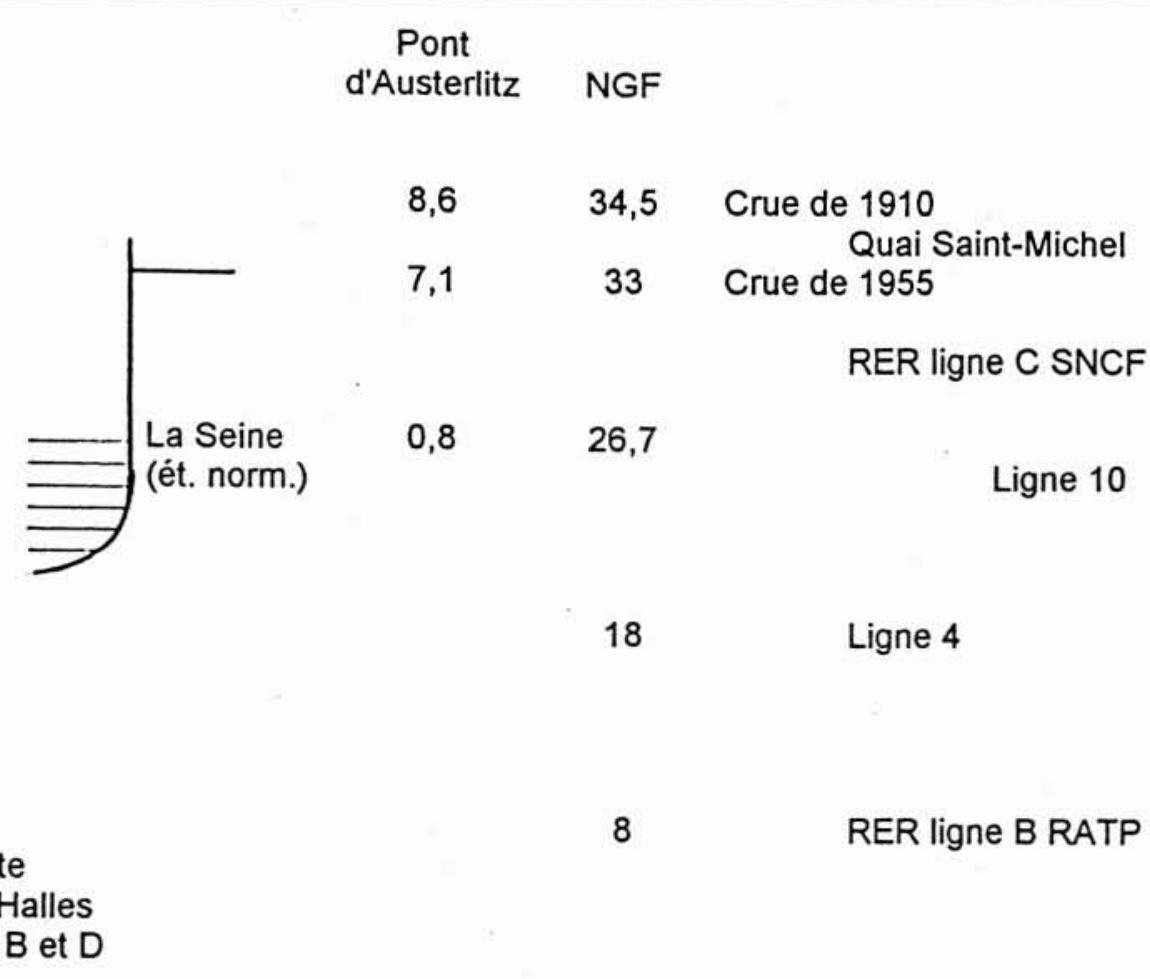

(Coupe perpendiculaire à la Seine, le nord à gauche)

4. Rive gauche : secteur Saint-Michel. 
tionnement de tout ce qui contribue à l'étanchéité des ouvrages ainsi qu'au drainage et au pompage corrélatif des eaux infiltrés. Lorsque la nappe remonte tant soit peu, on observe généralement la nécessité de parfaire à bref délai l'étanchéité en de nombreux points constitués notamment par des passages de câbles ou de canalisations dans les dalles et parois d'ouvrages ; progressivement ensuite, des portes étanches ainsi que des vannes sont fermées, des planchers en bois sont installés dans certains accès. Enfin, à partir d'un certain niveau atteint, ordre est donné d'édifier des murets et des barrages pour protéger contre le déversement toutes les trémies d'accès ou d'ouvrages établies sur la voie publique dans ses secteurs inondables.

Le plan comporte le descriptif de chaque muret ou barrage ainsi que la nature et la quantité des matériaux à approvisionner ; la liste des entreprises qui seraient appelées en urgence pour édifier les barrages est fréquemment mise à jour.

\subsection{Mesures complémentaires}

Sous réserve des vérifications actuellement effectuées, il est estimé que toutes ces mesures permettraient de protéger les réseaux si la hauteur d'eau ne dépassait pas 7,9 $\mathrm{m}$ à Paris-Austerlitz ; cependant, il serait opportun d'établir un état des besoins complémentaires en moyens mobiles de pompage et des entreprises de la région qui en disposent. De même, en raison de l'acccroissement sensible, né de l'extension des réseaux souterrains depuis les années 60 , du nombre de barrages et murets à édifier et de la nécessaire qualité de leur construction, il conviendrait d'identifier, en liaison avec la direction régionale de l'équipement, parmi les entreprises de travaux publics et de bâtiment soumises aux dispositions concernant la défense, toutes celles établies en proche banlieue et aptes à intervenir au même moment avec les matériaux appropriés pour l'édification des barrages. 